\title{
Individualized cardiac resynchronization therapy: current status
}

This article was published in the following Dove Press journal:

Research Reports in Clinical Cardiology

7 November 2014

Number of times this article has been viewed

\author{
Martin H Ruwald ${ }^{1,2}$ \\ Niels E Bruun ${ }^{1,3}$ \\ 'Department of Cardiology, Gentofte \\ Hospital, Hellerup, Denmark; ${ }^{2}$ Heart \\ Research Follow-up Program, Division \\ of Cardiology, University of Rochester \\ Medical Center, Rochester, NY, USA; \\ ${ }^{3}$ Clinical Institute, Aalborg University, \\ Aalborg, Denmark
}

\begin{abstract}
Cardiac resynchronization therapy (CRT) has shown a substantial reduction in heart failure patient morbidity and mortality, with improvement in quality of life as well as symptoms. The therapy is, however, limited to approximately $10 \%-15 \%$ of heart failure patients and, typically, 30\% do not derive benefit from the device. For optimal outcomes with CRT, the correct selection of patients is of paramount importance. The first parameter is depressed left ventricular systolic function, and the second is a wide QRS complex. Different nuances among clinical trials have rendered guidelines pragmatic and compromising, but also conflicting and confusing. A large proportion of real-life CRTs are implanted in patients where the evidence for benefit is scarce or not present. Further, for optimal benefit, patients require evidence-directed medical therapy at maximal doses, effective placement of ventricular leads, and high biventricular pacing percentages, along with optimized atrioventricular (AV) and interventricular interval device programming. These items, as well as specific clinical characteristics, such as AV block and atrial fibrillation, in the context of CRT indications, are discussed. This review focuses on these issues to guide the clinician through guidelines, with an evidence-based update on the current status of CRT.
\end{abstract}

Keywords: Cardiac resynchronization therapy, prognosis, review, biventricular pacing, guideline, indications

\section{Introduction - clinical trials and development}

Approximately $2 \%$ of the adult population in developed countries has clinical heart failure (HF), increasing prevalence with age, to more than $10 \%$ in patients $>70$ years old. ${ }^{1,2}$ At least half of these patients have systolic HF with reduced left ventricular ejection fraction (LVEF) (ie, heart failure with reduced ejection fraction [HfrEF]). The mainstay of pharmacological treatment for HFrEF during the last 2-3 decades has been a combined treatment, with inhibitors of the renin-angiotensin system and blockers of the beta-adrenergic and aldosterone receptors, which has reduced morbidity and mortality significantly. ${ }^{1}$ Although medical management has been successful in approximately $10 \%-15 \%$ of all HF patients, further patients have electrical conduction abnormalities and continuously depressed systolic function. ${ }^{3,4}$ Cardiac resynchronization therapy (CRT) has developed as a device-based treatment option available for patients with drug-refractory, mild, moderate, or severe heart failure. This device treatment modality has been shown to improve morbidity and mortality significantly, and has been confirmed in recent meta-analyses, but the therapy has so far been limited only to patients with depressed LVEF and specific electrical activation disturbances. ${ }^{5-14}$ The recent European CRT survey ${ }^{15}$ showed, however, that the CRT indications used in
Correspondence: Martin H Ruwald Heart Research Follow-up Program, Division of Cardiology, University of Rochester Medical Center, 265 Crittenden Boulevard, Rochester I 4642 NY, USA

Email mruwald@hotmail.com 
daily clinical practice went beyond what was recommended based on the landmark clinical trials, including implantation of the device in patients without conduction disturbances and in patients with permanent atrial fibrillation.

Landmark studies were initiated in the late 1990s and first evaluated the use of a CRT pacemaker (CRT-P) in moderate to severe HF patients (New York Heart Association [NYHA] classes III and Ambulatory IV). The MUSTIC, ${ }^{16}$ MIRACLE, ${ }^{8}$ and PATH-CHF ${ }^{17}$ trials demonstrated, in 2001 and 2002, short-term effects, with improvements in walking distance, quality of life, and NYHA class with the use of CRT-P, compared to optimal medical therapy. In 2003 and 2004, the CONTAK-CD ${ }^{18}$ and MIRACLE ICD I ${ }^{19}$ and $\mathrm{II}^{20}$ trials compared change to CRT with a defibrillator (CRT-D) to implantable cardioverter defibrillator (ICD) and found improved oxygen uptake, improved quality of life, walking distance, and NYHA class; and also during short-term follow-up for patients in NYHA classes ranging from II-IV. The COMPANION ${ }^{5}$ and CARE-HF ${ }^{6}$ trials from 2004 and 2005 provided the substance of long-term evidence determining the efficacy of CRT-P for NYHA III and Ambulatory IV, with significant reductions in mortality and all-cause or cardiovascular hospitalizations. Further, COMPANION had a third arm, randomized to use CRT-D, that was also associated with improved outcome, but there was insufficient statistical significance to show any additional benefit, as compared to CRT-P, and the trial was not designed for this. In 2008, the REVERSE ${ }^{21}$ trial indicated improvement in HF clinical composite score for mildly symptomatic NYHA class I and II patients in CRT-D versus ICD, and in 2009, the MADIT-CRT ${ }^{7}$ trial showed significant reductions in a combined end point of HF hospitalizations and all-cause mortality in patients with NYHA I or II symptoms. These were followed in 2010 by the RAFT $^{9}$ trial that compared CRT-D to ICD in NYHA class IIIII, and confirmed benefit by reductions in HF hospitalization or all-cause death in CRT-D. Long-term follow-up (7 years) of MADIT-CRT ${ }^{22}$ was recently published, showing significant reduction in all-cause mortality for CRT-D, compared to ICD, while long-term results from $\mathrm{REVERSE}^{23}$ found a very low yearly mortality rate $(2.9 \%)$ in patients treated with CRT-D. A meta-analysis ${ }^{13}$ found a relative mortality risk of 0.73 95\% Confidence intervals (CI: $0.62-0.85$ ) for CRT-P versus control (medications) and a relative risk of $0.78,95 \%$ Confidence intervals (CI: $0.70-0.87$ ) for CRT-D versus ICD. The number of patients needed to be treated with a CRT-D device for a duration of 7 years, to save one life (who had left bundle branch block [LBBB]), was nine, in the long-term follow-up from MADIT-CRT. ${ }^{22}$ A table of the randomized, clinical landmark trials is shown in Table 1. The evidence from these trials result in minor differences of opinion in interpretation of both LVEF and QRS duration in current Canadian, ${ }^{24} \mathrm{US},{ }^{25}$ and European guidelines, ${ }^{11}$ but a simple and practical synthesis is that CRT is highly recommended and beneficial in patients with sinus rhythm, LVEF $\leq 35 \%$, NYHA class II-ambulatory IV, and LBBB QRS pattern.

\section{Current status}

CRT is highly recommended and beneficial in patients with sinus rhythm, LVEF $\leq 35 \%$, NYHA class II-Ambulatory IV, and LBBB QRS pattern.

\section{Electrical dyssynchrony - the electrocardiogram}

All above-mentioned major CRT trials included patients with various degrees of prolonged QRS duration (a minimum of $120 \mathrm{~ms}$ ). The LESSER-EARTH ${ }^{26}$ trial, published in 2013, sought to examine the usefulness of CRT in patients with QRS $<120 \mathrm{~ms}$; it was terminated because of futility, and found that CRT did not improve clinical outcomes or left ventricular remodeling, and was associated with potential harm.

Among patients enrolled in the major trials, QRS morphology and duration have consistently been scrutinized to optimize the selection of patients. In MADIT-CRT, by original, prespecified subgroup analysis, the benefit of CRT was driven by a $41 \%$ reduction in HF or death, in patients with $\mathrm{QRS} \geq 150 \mathrm{~ms}$. A significant interaction between QRS morphology and CRT intervention was, however, reported in a subanalysis, ${ }^{27}$ revealing that only the $\mathrm{LBBB}$ patients benefited from CRT (53\% reduction in HF or death), while both right bundle branch block (RBBB) and intraventricular conduction delay (IVCD) did not achieve any benefit. These findings are largely caused by the significant overlap between QRS $>150 \mathrm{~ms}$ and LBBB QRS morphology, in 77\% of the patients of the MADIT-CRT study population. Two recent meta-analyses ${ }^{28,29}$ (based on MADIT-CRT, COMPANION, CARE-HF, REVERSE, and RAFT) examined the benefit by QRS morphology and by QRS duration. It was confirmed that benefit of CRT was only seen in LBBB patients, but when ignoring QRS morphology as a factor, a benefit was still seen in QRS $\geq 150 \mathrm{~ms}$. A retrospective analysis from $2010(n=14,946)^{30}$ reported that nearly one-third of patients implanted with CRT had non-LBBB, which was associated with increased mortality when compared to LBBB. These findings were supported by a later observational analysis in $2013^{31}$ $(n=24,169$; mean age: 75 years $)$ that reported 3 -year mortality 


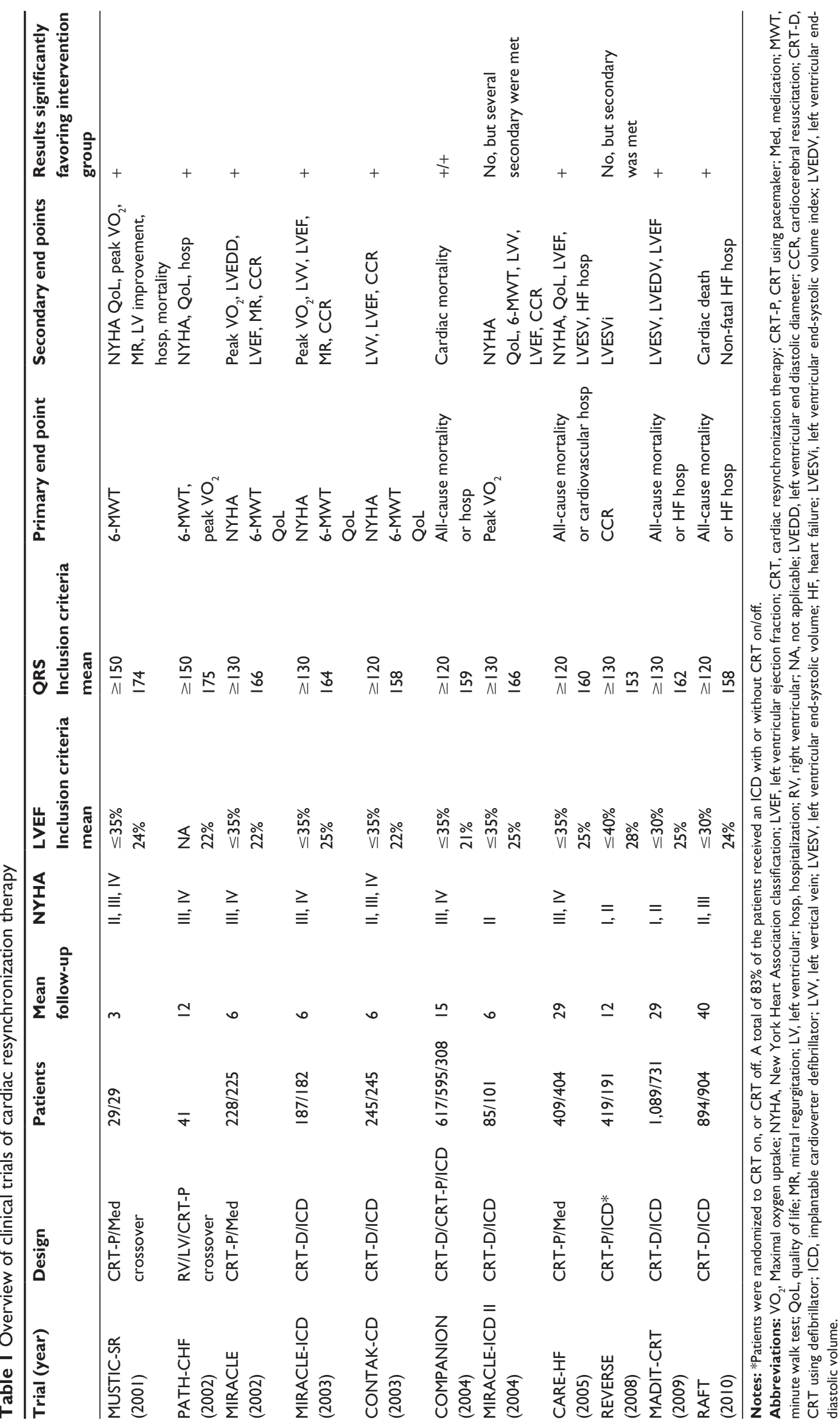


by duration and QRS morphology: LBBB QRS $\geq 150 \mathrm{~ms}$ of $20.9 \%$ (used as reference), LBBB QRS $120-149 \mathrm{~ms}$ of 26.5\% (hazard ratio [HR]: 1.30 [1.18-1.42]), non-LBBB QRS $\geq 150 \mathrm{~ms}$ of $30.7 \%$ (HR: 1.34 [1.20-1.49]), non-LBBB QRS $120-149$ ms of $32.3 \%$ (HR: 1.52 [1.38-1.67]). These two retrospective analyses did not have control groups and therefore, as such, could not be used to show the efficacy of CRT-D versus ICD, but outlined the relative risks and mortality by both QRS morphology and duration, in large, real-life cohorts of patients. Recently, however, an individual-level patient metaanalysis $^{14}$ (from CARE-HF, MIRACLE, MIRACLE ICD, REVERSE, and RAFT) reported benefit of CRT at $140 \mathrm{~ms}$ and above. In this study, a multivariable model suggested that only QRS duration predicted the effect of CRT on outcomes and therefore, in this study, QRS morphology alone was not a determinant factor. The battle between QRS duration versus QRS morphology as the determinant factor thus continues. In LBBB, the electrical activation sequence initiates in the right ventricular (RV) wall and slowly progresses through the septal wall to the left ventricular (LV) apex and lateral wall. In essence, LBBB QRS morphology reflects a pathophysiological electrical substrate that is highly amenable to CRT, while it also makes sense that there seems to be no beneficial effect of LV pacing in RBBB patients.

The above-reported evidence results in various interpretations of the recommendation of QRS duration in current Canadian, ${ }^{24} \mathrm{US},{ }^{25}$ and European guidelines. ${ }^{11} \mathrm{~A}$ practical approach is that CRT is recommended and beneficial in patients with LBBB QRS pattern, independent of QRS duration when above $120 \mathrm{~ms}$, and it is unlikely future trials will change this. For an LBBB pattern with QRS $>150 \mathrm{~ms}$, the patients experience even more pronounced CRT benefit and therefore, this configuration received benficial (IA) indication. A non-LBBB pattern with $\mathrm{QRS} \geq 150 \mathrm{~ms}$ received "should be considered" (IIa B) in NYHA II, III, and IV, in Europe; "should be considered" (IIa A) in NYHA III and IV, and "may be considered" (IIb B) for NYHA II, in the US; and finally, a "may be considered" (IIb-low quality) in Canadian guidelines. A non-LBBB 120-149 ms received "may be considered" (IIb) in US and European guidelines but, importantly, there is no actual evidence to suggest any benefit in non-LBBB patients with QRS $<150 \mathrm{~ms}$. Finally, there is currently consensus that CRT should not be implanted in patients with QRS $<120 \mathrm{~ms}$.

\section{Current status}

CRT is recommended and beneficial in patients with LBBB, regardless of QRS duration when above $120 \mathrm{~ms}$, with a pronounced effect in QRS $>150 \mathrm{~ms}$. Non-LBBB patients with QRS $\geq 150$ ms may benefit and non-LBBB with QRS $<150$ ms likely may not benefit. CRT in patients with QRS $<120 \mathrm{~ms}$ is not recommended.

\section{Mechanical dyssynchrony - the echocardiogram}

In the last 10 years, echocardiographic methods to better characterize the underlying electrical and regional mechanical function have evolved. ${ }^{32}$ Intraventricular systolic (mechanical) dyssynchrony refers to differences in the timing of contraction between the different myocardial segments. Traditionally, dyssynchrony has been defined as an increased time delay between the peak of shortening of tissue velocity between LV free wall and septal wall (annotated time-to-peak), and several definitions and measurements exist by M-mode, tissue Doppler imaging, and speckle-tracking radial and longitudinal strain. The rationale in CRT was that dyssynchrony measurements could provide additional information to the clinician for correct selection of patients for CRT, beyond QRS width. There is, however, no consensus on how ventricular dyssynchrony should be measured, nor has any definition yet been proven robust, ${ }^{33,34}$ and the search for simple and reproducible echocardiographic dyssynchrony measures to predict beneficial outcome of CRT is ongoing. ${ }^{35}$ Practically, the echocardiographic CRT studies can be divided into $\mathrm{QRS} \geq 120 \mathrm{~ms}$ and $\mathrm{QRS}<120 \mathrm{~ms}$.

The PROSPECT ${ }^{33}$ study of 426 patients with wide QRS failed to show any use of echocardiographic dyssynchrony measurements in the evaluation of CRT response, but this study was criticized for a number of technical shortcomings. Technical differences between echocardiography centers, vendors, and a variability in interpretation of measurements introduced confounding variables that likely affected the negative PROSPECT study results. ${ }^{36}$ Thus, it is currently unknown if a specific reproducible measurement of mechanical dyssynchrony can provide additional selection of patients with QRS $>120$ ms specifically among non-LBBB. With implantation of many CRT devices, particularly in non-LBBB patients, a correct dyssynchrony-targeted approach may have the potential to improve the selection of CRT patients in the wide QRS HF population. ${ }^{35}$ Tissue-Doppler cross-correlation analysis may help answer this issue, but prospective studies are needed. ${ }^{35}$

In patients with narrow QRS, some single-center studies suggested that echocardiographic dyssynchrony criteria could identify patients who would respond to CRT. ${ }^{37,38}$ The randomized RethinQ ${ }^{39}$ trial from 2007 included 172 patients with 
QRS $<120 \mathrm{~ms}$ and significant intraventricular mechanical delay. However, it found no beneficial effects of CRT, compared to ICD, as measured by 6-month HF events and peak oxygen consumption. These results were subsequently confirmed in the larger and definitive outcome trial of Echo-CRT ${ }^{40}$ that was stopped in 2013, for futility. The study included 809 patients and, during an average follow-up of 19 months, found that CRT did not reduce death or HF hospitalization and could even be associated with increased mortality, with $11 \%$ deaths in the CRT group versus $6 \%$ in the $\operatorname{ICD}$ group $(P=0.02)$. These results, along with the negative results from the LESSER-EARTH, in a sense, concluded that ECG dyssynchrony, wide QRS complex, and in particular, LBBB are excellent electric markers for identification of HF patients who are likely to benefit from CRT, while so far, patients with narrow QRS, mechanical dyssynchrony, or not, do not benefit from CRT. Finally echocardiographic imaging, of course, remains a valuable clinical assessment of the non-responder post-CRT implantation, for individual optimization of atrioventricular (AV) and interventricular (VV) dyssynchrony, as described below.

\section{Current status}

The role of echocardiographic-measured dyssynchrony to select CRT candidates has not been fully determined. There may be a role for speckle tracking to improve selection of candidates, specifically with wide complex QRS non-LBBB, while it currently cannot be used to guide CRT for dyssynchronous, narrow QRS complex patients.

\section{Myocardial substrate - specific clinical characteristics and patient comorbidities}

Despite a significant number of patients having both permanent atrial fibrillation (AF) and HF, these patients have not been included in the main trials. More than $20 \%$ of CRT recipients in Europe have permanent $A F,{ }^{15}$ and these patients do not achieve optimal benefit. Studies comparing sinus rhythm patients to permanent AF patients have consistently showed a reduced efficacy and increased rate of nonresponders among AF patients. This was shown in a recent meta-analysis ${ }^{41}$ compiling 23 of the observational studies $(n=7,495)$, of which $25.5 \%$ had AF. Patients with AF had $32 \%$ increased risk of being a non-responder and a $50 \%$ increased mortality rate. The RAFT ${ }^{9}$ trial included $13 \%$ permanent AF patients and this subgroup did not show any benefit from CRT-D, as compared to ICD. On the other hand, the MUSTIC $\mathrm{AF}^{42}(\mathrm{n}=59)$ trial indicated that CRT (compared to RV pac- ing) could be beneficial in permanent, slow AF patients with wide QRS and NYHA III, when evaluating those achieving $>85 \%$ biventricular (BIV) pacing. The $\mathrm{APAF}^{43}(\mathrm{n}=186)$ and PAVE $^{44}(n=184)$ studies further showed that AV nodal ablation and CRT improved symptoms and reduced hospitalizations, compared to RV pacing only, and implantation of CRT in AF patients is now a class IIa B recommendation in European guidelines. ${ }^{11}$ The large CERTIFY registry ${ }^{45}$ $(n=7,384)$ found no difference in mortality between CRT patients with sinus rhythm and AF patients with AV nodal ablation, while AF patients treated with drugs only had significantly higher mortality, essentially advocating a higher level of recommendation for AV nodal ablation in AF patients undergoing CRT.

These considerations all concerned permanent AF, but it is undetermined what influence persistent or paroxysmal AF plays for CRT efficacy. A recent substudy from MADIT-CRT $^{46}$ showed that prior or in-trial paroxysmal or intermittent AF did not affect CRT outcomes, indicating that only permanent AF (and/or if BIV pacing percentages are substantially reduced) affects the long-term clinical outcome. No randomized trial has, however, shown mortality benefit associated with AV nodal ablation for permanent AF patients, and the potential benefits of CRT must be balanced against the risks of performing AV nodal ablation, including perioperative infections and pacemaker dependency.

The PREVENT-HF ${ }^{47}$ trial $(n=108)$ compared CRT to RV pacing in patients with no overt HF and AV block, and found no benefit and an insignificant trend towards reduction in HF events. This was somewhat in contrast to the PACE study ${ }^{48}(n=177)$, in which a preservation of LVEF was demonstrated in patients receiving a CRT, whereas RV pacing only led to a reduction in LVEF and an increase in left ventricular end-systolic volume (LVESV). Just recently, the BLOCK-HF ${ }^{49}$ ( $\left.n=691\right)$, however, further expanded CRT indications, evaluating patients with AV conduction disturbances and LVEF $<50 \%$. The study showed a $28 \%$ reduction in a combined end point of mortality, HF events, and increase in LVESV, comparing CRT to RV pacing. The results of the BioPace trial, ${ }^{50,51}$ including more than 1,800 patients with similar characteristics as PREVENT-HF and BLOCK-HF, is underway and will help shed light on the indications for CRT in this population.

\section{Current status}

CRT in permanent AF patients should be considered in patients with severe HF and QRS $>120 \mathrm{~ms}$ or with reduced LVEF if AV nodal ablation is performed in patients with 
incomplete BIV pacing, or with incomplete rate control. CRT may reduce HF events and symptoms in patients with a traditional bradycardia indication for RV pacing and moderately reduced LVEF, but at the cost of a higher complication rate.

\section{Device details - lead positions}

There is a substantial variability in what determines an optimal LV lead position. LV lead implantation is limited by constraints of the venous anatomy, phrenic nerve pacing, lead stability, and pacing threshold. Most implanting physicians battle these impediments and struggle, often unsuccessfully, to place the lead along the posterolateral and lateral walls. In MADIT-CRT ${ }^{52}$ and REVERSE, ${ }^{53}$ an LV lead position in the apical region was associated with adverse outcome, while an anterior position was associated with increased risk of ventricular arrhythmias. ${ }^{54}$ Thus, leads are, a priori, best placed in the non-apical region. The TARGET ${ }^{55}$ trial showed that speckle tracking-guided LV lead positioning can further improve LV reverse remodeling, as compared to standard LV lead placement, in LBBB patients. Similar and confirmatory results were found in the STARTER ${ }^{56}$ trial, including 110 patients with echo-guided lead placement and 77 controls with standard lateral or posterior LV lead sites. This approach improved patient outcomes by reducing the combined risk of death or HF hospitalizations (HR: 0.48; $P=0.006$ ). The study included patients with $\mathrm{QRS} \geq 120 \mathrm{~ms}$, but information concerning QRS morphology was not provided. Results of a smaller trial, IMAGING-CRT, ${ }^{57}$ that uses a multimodality imaging approach, is underway but, again, only in LBBB patients where CRT responder rate is already known to be very good and even as high as $91 \%$ in MADIT-CRT. ${ }^{58}$

The individual parameters proposed to predict response to CRT, including LV dyssynchrony by strain imaging, ${ }^{32}$ cross correlation analysis, ${ }^{35}$ the optimal LV lead position, ${ }^{59}$ and myocardial scar burden ${ }^{60}$ may, in combination, add prognostic value, ${ }^{61,62}$ but, so far, no definitive comparative studies have been able to select any one of these to be included in preselection of CRT candidates. The super-response of many LBBB patients is most likely due to the presence of a large anatomic sweet spot of potential response, particularly in non-ischemic patients. Probably, the exact LV lead placement would matter more in non-LBBB and non-responder subgroup populations, such as ischemic patients (even with LBBB), if therapy were guided by imaging; but, importantly, the anatomy of the venous system remains the single most important limitation to obtaining this. Thus, it remains still to determine which non-LBBB patients are likely to respond, and prospective lead placement-guided CRT trials are needed in this population.

\section{Current status}

LV lead position should be placed in the non-apical region. The role of echo-guided placement is still undetermined and appears unnecessary for non-ischemic patients with wide QRS, but it could play an important future role among ischemic patients, with or without LBBB, for optimal response.

\section{Device interrogation and programming}

Optimal response to CRT depends on the ability of the device to deliver as close to $100 \%$ biventricular (BIV) pacing as possible. Effective delivery of CRT may be hindered by the presence of native ventricular conduction, by inappropriate long AV delay programming, by atrial or ventricular tachycardia, atrial fibrillation, or frequent premature atrial and ventricular complexes.

The optimal BIV pacing percentage has been evaluated in two retrospective studies of 1,812 patients, by Koplan et al, ${ }^{63}$ and 36,935 patients, from the ALTITUDE study by Hayes et al. ${ }^{64}$ Koplan et al found 92\%, while Hayes et al found $98.5 \%$ to be cut-offs, with the greatest magnitude of separation for total mortality. In particular, Hayes et al found that patients with AF had similar survival as sinus rhythm patients, as long as they achieved BIV pacing percent of $98.5 \%$ or more. Finally, the MADIT CRT study ${ }^{65}$ found a cut-off of $97 \%$ to separate risks of HF events and death for CRT patients, while $>90 \%$ defined the cut-off of CRT efficacy, when compared to ICD-only. ${ }^{65}$

A typical mechanism that leads to a reduction in BIV pacing is loss of LV pacing due to dislodgment or lack of capture of the LV lead. A second mechanism is an intrinsic conduction, faster than the programmed AV delay. A third important mechanism is atrial arrhythmias with native conducted beats and ventricular arrhythmias interfering with CRT delivery. Recently, it was shown that Holtermeasured preimplantation atrial and ventricular premature beats significantly reduces the chance of high BIV pacing percentage (above 97\%), and patients with $0.1 \%-1.5 \%$ and $>1.5 \%$ ectopic beats were associated with adverse outcome, as compared to those with $<0.1 \%$ ectopic beats. ${ }^{66}$ Several approaches have been proposed to optimize the AV and VV delays, but no clear difference between automatic electrocardiographic algorithms and echocardiographic CRT optimization has been found in larger randomized trials, such as the SMART-AV trial $(n=1014)^{67}$ and the 
Adaptive CRT trial $(\mathrm{n}=522) .{ }^{68}$ The Adaptive CRT trial ${ }^{68}$ evaluated an automatic algorithm, adjusting the CRT pacing delivery by providing an automatic ambulatory dynamic optimization of AV and VV delays. Currently echocardiographic AV and VV optimization of all patients, versus a fixed AV delay of 100-120 ms, results in limited benefit, while for the non-responder, echocardiographic AV and VV optimization remains an important follow-up measurement and control parameter. Acute hemodynamic changes can be echocardiographically monitored, on a beat-to-beat basis, by the initiation or cessation of LV pacing. Several individual echocardiography Doppler parameters have been used for optimization of CRT. ${ }^{69,70}$ One method for AV delay optimization, recommended in guidelines, is by Doppler echocardiography of transmitral flow, using the iterative method (Figure 1). ${ }^{71}$ The AV delay found to provide maxi- mum separation of the E and A waves can be programmed, but this method has, however, been criticized for high inter- and intraobserver variability. ${ }^{72} \mathrm{VV}$ optimization can be performed by a number of estimations of stroke volume. ${ }^{71,73-75}$ Non-randomized studies have shown benefit of VV optimization, and improved exercise capacity, ${ }^{74,76}$ while randomized studies ${ }^{77,78}$ have failed to show an effect, when compared to a nominal setting. In clinical practice, however, many patients do not undergo the recommended echocardiographic optimization process, because of the lack of availability of skilled staff time. This may explain why optimization is low and only $45 \%$ of patients received AV delay optimization in a recent survey. ${ }^{79}$ Optimization is likely to remain low until a more technically convenient method is available, and since measurement of the CRT effect is of similar size as the variability of the measured

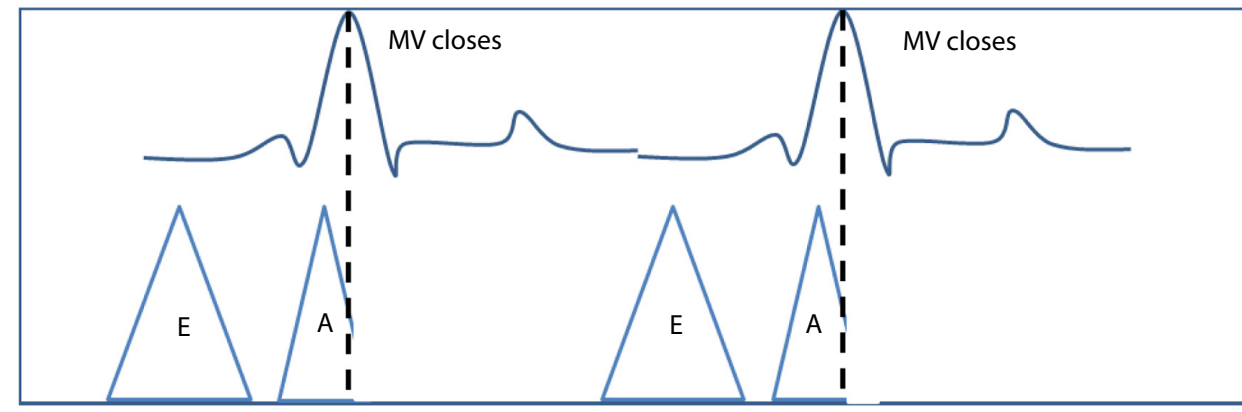

The AV delay is too short. The atria do not fully contribute to ventricular filling because of early closing of MV. The A wave is truncated when measuring the mitral inflow by pulsed wave doppler.

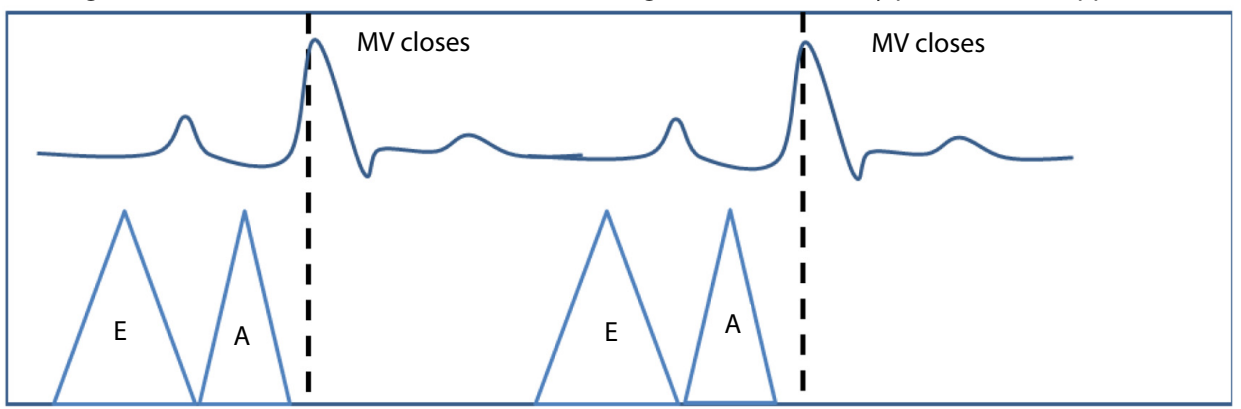

The AV delay is adequate. No truncation of $A$ wave and $E$ and $A$ wave are separated.

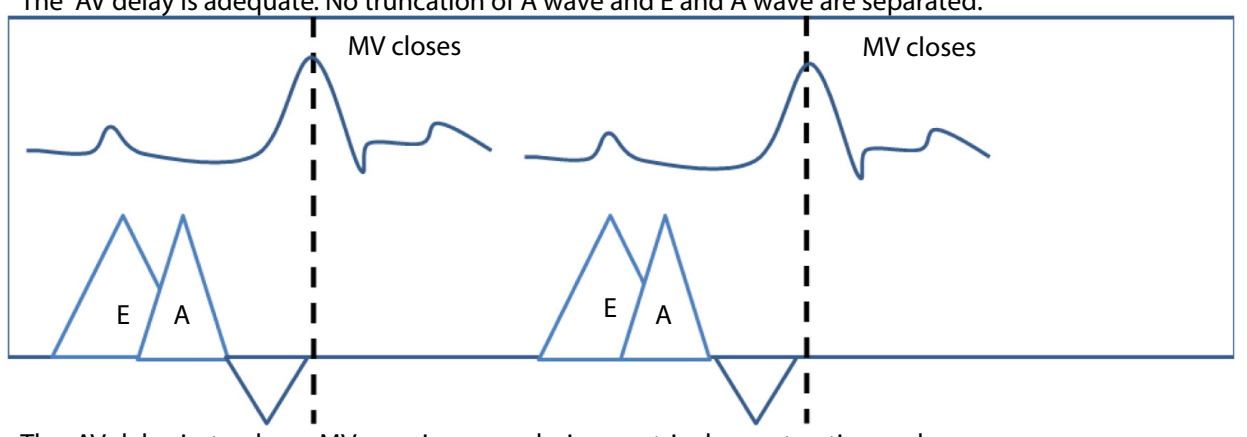

The AV delay is too long. MV remains open during ventricular contraction and presystolic mitral regurgitation is present

Figure I Echocardiographic optimization of atrioventricular delay using the iterative method. Abbreviations: $\mathrm{AV}$, atrioventricular; $\mathrm{MV}$, mitral valve. 
echocardiographic parameter, a true effect is difficult to evaluate. Among studies underway to further define this area is the BRAVO study, ${ }^{80}$ which is a noninferiority trial, comparing echocardiographic optimization of AV and VV delay with simple, noninvasive blood pressure monitoring.

\section{Current status}

Correct AV delay programming is essential for optimal CRT response. BIV pacing percentage as close to $100 \%$ as possible should be a goal, and frequent interrogation and interpretation of a declining BIV pace percentage should result in optimization measures, such as AV nodal and ventricular premature complexes, antiarrhythmic drug prescriptions, and device parameter (AV and VV delays) reprogramming. Holter monitoring and echocardiograms are essential in the evaluation.

\section{Pharmacological considerations}

Since the early CRT trials, there has been continuously increasing use of evidence-based medications concomitantly with CRT implantations, with frequencies of angiotensin receptor blockers/angiotensin converting enzyme inhibitors of 89\%-90\% in COMPANION (2004), to 96\%-97\% in RAFT (2010); beta blockers of 6\% in COMPANION, to 70\%-74\% in CARE-HF (2005), to 93\% in MADIT-CRT (2009); and mineralocorticoid receptor antagonists from 3\%-5\% in COMPANION, to $54 \%-59 \%$ in CARE-HF, and, naturally, somewhat lower in the lower NYHA studies: $31 \%-32 \%$ in MADIT-CRT and $42 \%$ in RAFT. Patients were typically on "optimal" guideline medications at time of implantation, but several subanalyses have shown that this was not the case with dosages. In fact, CRT has been associated with improved outcome in those on increased dosages, and less likelihood of being treated with diuretics, particularly in responders. ${ }^{81-86}$ Whether or not this is because of increased neurohormonal blockade before CRT treatment or a result of the response to CRT allowing an increase in the tolerable dosages of these medications is not clear, and additional data are needed. Recent subanalysis from MADIT-CRT ${ }^{86,87}$ showed that patients on carvedilol had improved outcome, compared to patients on metoprolol (HR: $0.70 ; P=0.001$ ), more pronounced in CRT-D (HR: $0.61 ; P=0.001$ ), and in CRT-D with LBBB (HR: 0.51 ; $P<0.001)$. Throughout the trial, an increase in dosages was seen, and there was a dose-dependent relationship with outcomes found for carvedilol, but not metoprolol.

In short, all patients should be on optimized, fully-titrated, evidence-based HF medications at time of CRT implantation, and if not the case, it is important that those who respond are evaluated for further titration.

\section{Current status}

Patients should be on optimal HF medication therapy with evidence-based, derived maximum doses. If not, it is likely CRT can further enhance the chance of a further titration to maximal doses, particularly among super-responders.

\section{Optimal responders versus classical non-responders}

Several analyses have consistently shown that patients with LBBB QRS morphology (and QRS >150 ms, as discussed above), with non-ischemic cardiomyopathy, less scar burden, females, and reduced disease burden (smaller atrial and ventricular volumes and higher baseline LVEF) derive a relatively greater benefit of CRT implantation. Figure 2 shows the typical features identifying classical responders and non-responders. The chance of response (as measured by $>15 \%$ decrease in LVESV) is typically reported to be around $70 \%$, based on the historical CRT populations with less strict QRS morphology and QRS width inclusion criteria. Simple selection of patients may increase this number substantially; alone in the MADIT-CRT, ${ }^{58}$ a 91\% responder rate is achieved for all LBBB patients. Suboptimal response to CRT is multifactorial, thus relying on patient selection and CRT delivery techniques (AV delay, lead positions etc). Mullens et al ${ }^{88,89}$ showed that a protocol-driven assessment of CRT non-responders improves reverse remodeling by $50 \%$, and a $38 \%$ reduction in clinical outcomes has been shown in a retrospective analysis from a multidisciplinary

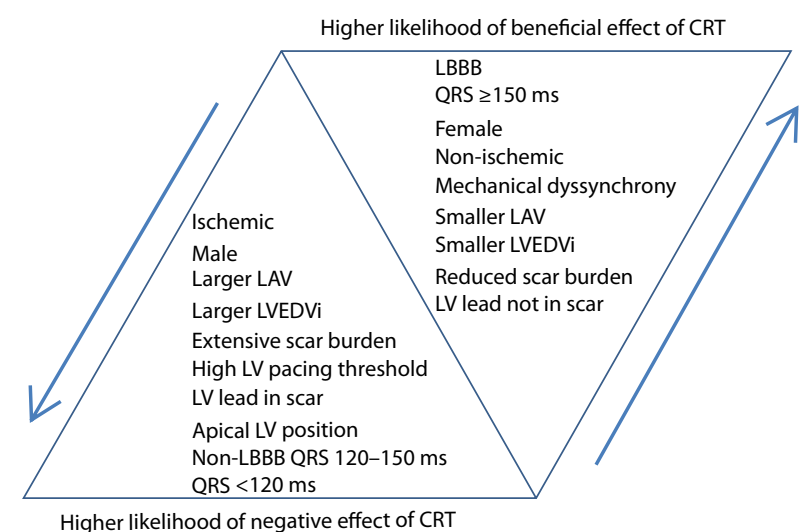

Figure 2 Chance of successful CRT outcome by patient characteristics: factors that increase or decrease the likelihood of beneficial effect or cardiac resynchronization therapy.

Abbreviations: CRT, cardiac resynchronization therapy; LBBB, left bundle branch block; LAV, left atrial volume; LVEDVi, left ventricular end-systolic volume index; LV, left ventricular. 


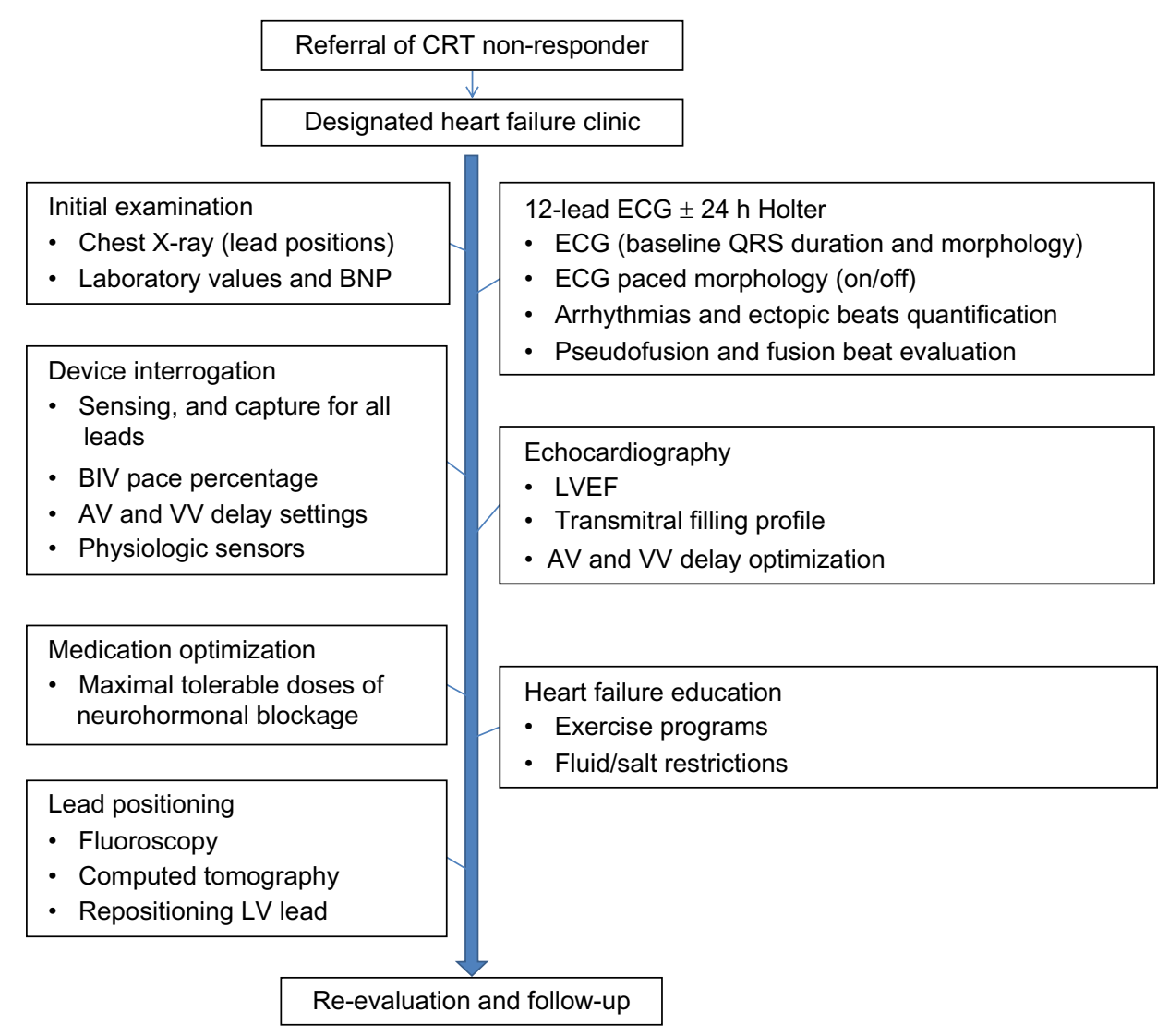

Figure 3 Stepwise assessment of a non-responder to cardiac resynchronization therapy.

Notes: Management of the cardiac resynchronization therapy non-responder is a multimodality effort, with involvement of many diagnostic processes and procedures. Abbreviations: CRT, cardiac resynchronization therapy; BNP, brain-type natriuretic peptide; ECG, electrocardiogram; BIV, biventricular; AV, atrioventricular; VV, interventricular; LVEF, left ventricular ejection fraction; LV, left ventricular.

care setting, compared to "conventional" care. ${ }^{90}$ Figure 3 shows a practical approach for evaluation, assessment, and optimization of the non-responder.

\section{CRT-P versus CRT-D}

There is a wide variation in the use of CRT devices in individual countries. In the United States, CRT-D was used in over $80 \%$ of patients implanted with CRT devices in $2004,{ }^{91}$ while in Europe, the use of CRT-D ranged from 9\% to $92 \%$ (median 45\%). ${ }^{92}$ The decision to include ICD backup for patients suitable for CRT has significant implications in terms of complications, inappropriate ICD therapies, health care costs, and cost-effectiveness ratio. ${ }^{11,93}$

Should ICD backup be added routinely to all patients considered suitable for CRT implantation? The case for CRT-D is good for secondary prevention of sudden cardiac death (SCD) by potentially-treatable ventricular arrhythmias, and SCD still accounts for about one-third of all deaths in patients treated with CRT-P., Many patients who are suitable for CRT also fulfill present indications for ICD. ${ }^{25}$ It may therefore be considered safer to implant a combined device to further reduce SCD. The survival benefit favoring CRT-D is, however, discussable, particularly since the evidence from COMPANION, ${ }^{5}$ as well as a meta-analysis,${ }^{94}$ were unable to show this. The COMPANION 5 study indicated that a possible incremental benefit of ICD may be short-lived, since survival curves between CRT-D and CRT-P were parallel after 9 months of therapy. Furthermore, CRT-P induces reverse remodeling and improves left ventricular function, which potentially reduces the risk of subsequent SCD by a reduction in risk of ventricular arrhythmias. ${ }^{95}$ This is consistent with data from CARE-HF, ${ }^{6}$ suggesting that CRT-P alone reduces SCD, as well as total mortality. Recent data from MADIT-CRT ${ }^{96}$ further showed that, among those who experience a normalization of $\operatorname{LVEF}(>50 \%)$, the absolute and relative risks of ventricular arrhythmias are very low, while for those who recover LVEF to $36 \%-50 \%$, ventricular arrhythmias remain a substantial issue. In MADIT-II, ${ }^{97}$ risk factors such as age $>70$, NYHA $>$ II, reduced kidney function, QRS $>120 \mathrm{~ms}$, and AF were reported (in ischemic patients), where the benefit of ICD (compared to medical therapy) was attenuated if more than three factors were present. Further, a reduced benefit was 
shown in NYHA III patients (ischemic and nonischemic) in the SCD-HeFT trial..$^{98}$ Overall factors favoring CRT-P, besides lower cost and lower complication rate, are, therefore, more advanced HF patients, very elderly, severe renal insufficiency or other major comorbidities, and life expectancy less than 1 year. The group of patients with no prior ventricular arrhythmias, and those who are very likely to have major reverse remodeling or LVEF normalization (non-ischemic, baseline LVEF > 30\%-35\%, females, LBBB QRS morphology $)^{99}$ (Figure 2) may not need the defibrillator, but this needs to be established in larger trials, and data is needed. The DANISH study (clinicaltrials.org: NCT00542945) ${ }^{100}$ may provide insights into the use of ICDs in nonischemic cardiomyopathy, with or without the biventricular pacemaker.

\section{Current status}

The potential incremental survival value of CRT-D, compared to CRT-P, has not yet been demonstrated. If both ICD and CRT indications are present, the choice is easy, with a resolute reduction in SCD. CRT-P alone may be the preferred choice in selected patients, where the risk of sudden cardiac death (as compared to non-sudden death) is low, or where the chance of future appropriate ICD utilization is very low, or the life expectancy is short.

\section{CRT complications}

Implantation of CRT is successful in most patients $(>90 \%)$ but is associated with a considerable rate of complications,

Table 2 30-day complication rates in CRT.

\begin{tabular}{ll}
\hline Implantation success rate & $93.8 \%-94.8 \%$ \\
Peri-implantation death & $0.2 \%-0.5 \%$ \\
Mechanical complications & $2.8 \%-3.6 \%$ \\
$\quad$ Coronary sinus dissection & \\
$\quad$ Pericardial effusion/tamponade & \\
Pneumothorax & \\
Hemothorax & $5.6 \%-6.8 \%$ \\
Lead problems & \\
Arrhythmias & \\
Dislodgements & \\
Dispositioned & \\
Diaphragm stimulation & $1.5 \%-2.4 \%$ \\
Device malfunctions & \\
Inappropriate shocks & \\
Sensitivity or pacing threshold issues & $2.9 \%-9.5 \%$ \\
Hematomas & $2.5 \%$ \\
Evacuation needed & $1.1 \%-1.7 \%$ \\
Infections & $11 \%-14 \%$ \\
Overall complication rate (excluding technical implant \\
failures)
\end{tabular}

Abbreviations: CRT, cardiac resynchronization therapy; ref, reference. according to large recent meta-analyses. ${ }^{101,102}$ The typical 30-day complication rates are shown in Table 2. Most complications occur in hospital or during the first 6 months, ${ }^{103}$ and lower volumes of implanting center and individual operators are major determinants for higher complication rates. ${ }^{104-106}$ The analysis by Adabag et al ${ }^{101}$ showed a total 30 -day adverse event rate (including the $6.6 \%$ of nonsuccessful implantations) of $18 \%$ for CRT implantation, versus $4 \%$ for ICD-only.

\section{Current status}

CRT implantation complication rates are relatively high, due to the complexity of the device and implantation procedure. These risks are outweighed by the great benefit of the device but require thoughtful balance of risk versus benefit in individual patients.

\section{Conclusion}

CRT has improved the clinical outcomes of selected HF patients significantly. However, considerable numbers of devices are being implanted in patients who do not meet conventional criteria, and even if strict criteria are used, a proportion of patients still do not respond to CRT. This review highlights the importance of patient selection and post-implantation CRT optimization, and discusses the contemporary clinical options available. The interplay of influencing factors is complex, and more research is needed for optimal usage of limited health care resources. Specific areas of research needed are within echocardiographic methods, or multi-modality imaging-guided LV lead placement, for non-LBBB patients, and for ischemic cardiomyopathy patients with large scar burden. Other areas to be defined in the near future are: indications for CRT in patients with permanent AF with AV nodal ablation, the use of CRT in nonLBBB $<150 \mathrm{~ms}$ and $\geq 150 \mathrm{~ms}$, the use of CRT in patients with more preserved LVEF, and specifying the optimal use of CRT-P versus CRT-D.

\section{Disclosure}

The authors report no conflicts of interest in this work.

\section{References}

1. McMurray JJ, Adamopoulos S, Anker SD, et al. ESC guidelines for the diagnosis and treatment of acute and chronic heart failure 2012: the Task Force for the Diagnosis and Treatment of Acute and Chronic Heart Failure 2012 of the European Society of Cardiology. Developed in collaboration with the Heart Failure Association (HFA) of the ESC. Eur J Heart Fail. 2012;14:803-869.

2. Mosterd A, Hoes AW. Clinical epidemiology of heart failure. Heart. 2007;93:1137-1146. 
3. Khan NK, Goode KM, Cleland JG, et al. Prevalence of ECG abnormalities in an international survey of patients with suspected or confirmed heart failure at death or discharge. Eur J Heart Fail. 2007;9:491-501.

4. Clark AL, Goode K, Cleland JG. The prevalence and incidence of left bundle branch block in ambulant patients with chronic heart failure. Eur J Heart Fail. 2008;10:696-702.

5. Bristow MR, Saxon LA, Boehmer J, et al. Cardiac-resynchronization therapy with or without an implantable defibrillator in advanced chronic heart failure. N Engl J Med. 2004;350:2140-2150.

6. Cleland JG, Daubert JC, Erdmann E, et al. The effect of cardiac resynchronization on morbidity and mortality in heart failure. $N$ Engl J Med 2005;352:1539-1549.

7. Moss AJ, Hall WJ, Cannom DS, et al. Cardiac-resynchronization therapy for the prevention of heart-failure events. $N$ Engl J Med. 2009;361:1329-1338.

8. Abraham WT, Fisher WG, Smith AL, et al. Cardiac resynchronization in chronic heart failure. $N$ Engl J Med. 2002;346:1845-1853.

9. Tang AS, Wells GA, Talajic M, et al. Cardiac-resynchronization therapy for mild-to-moderate heart failure. $N$ Engl J Med. 2010;363: 2385-2395.

10. Linde C, Abraham WT, Gold MR, Daubert C. Cardiac resynchronization therapy in asymptomatic or mildly symptomatic heart failure patients in relation to etiology: results from the REVERSE (resynchronization reverses remodeling in systolic left ventricular dysfunction) study. J Am Coll Cardiol. 2010;56:1826-1831.

11. Brignole M, Auricchio A, Baron-Esquivias G, et al. 2013 ESC guidelines on cardiac pacing and cardiac resynchronization therapy: the task force on cardiac pacing and resynchronization therapy of the European Society of Cardiology (ESC). Developed in collaboration with the European Heart Rhythm Association (EHRA). Eur Heart $J$. 2013;34:2281-2329.

12. Tracy CM, Epstein AE, Darbar D, et al. 2012 ACCF/AHA/HRS focused update incorporated into the ACCF/AHA/HRS 2008 guidelines for device-based therapy of cardiac rhythm abnormalities: a report of the American College of Cardiology Foundation/American Heart Association Task Force on Practice Guidelines and the Heart Rhythm Society. J Am Coll Cardiol. 2013;61:e6-e75.

13. Wells G, Parkash R, Healey JS, et al. Cardiac resynchronization therapy: A meta-analysis of randomized controlled trials. CMAJ. 2011;183:421-429.

14. Cleland JG, Abraham WT, Linde C, et al. An individual patient metaanalysis of five randomized trials assessing the effects of cardiac resynchronization therapy on morbidity and mortality in patients with symptomatic heart failure. Eur Heart J. 2013;34:3547-3556.

15. Bogale N, Priori S, Cleland JG, et al. The european CRT survey: 1 year (9-15 months) follow-up results. Eur J Heart Fail. 2012;14:61-73.

16. Cazeau S, Leclercq C, Lavergne T, et al. Effects of multisite biventricular pacing in patients with heart failure and intraventricular conduction delay. $N$ Engl J Med. 2001;344:873-880.

17. Auricchio A, Stellbrink C, Sack S, et al. Long-term clinical effect of hemodynamically optimized cardiac resynchronization therapy in patients with heart failure and ventricular conduction delay. $J$ Am Coll Cardiol. 2002;39:2026-2033.

18. Higgins SL, Hummel JD, Niazi IK, et al. Cardiac resynchronization therapy for the treatment of heart failure in patients with intraventricular conduction delay and malignant ventricular tachyarrhythmias. $J \mathrm{Am}$ Coll Cardiol. 2003;42:1454-1459.

19. Young JB, Abraham WT, Smith AL, et al. Combined cardiac resynchronization and implantable cardioversion defibrillation in advanced chronic heart failure: the MIRACLE ICD trial. JAMA. 2003;289:2685-2694.

20. Abraham WT, Young JB, Leon AR, et al. Effects of cardiac resynchronization on disease progression in patients with left ventricular systolic dysfunction, an indication for an implantable cardioverterdefibrillator, and mildly symptomatic chronic heart failure. Circulation. 2004;110:2864-2868.
21. Linde C, Abraham WT, Gold MR, et al. Randomized trial of cardiac resynchronization in mildly symptomatic heart failure patients and in asymptomatic patients with left ventricular dysfunction and previous heart failure symptoms. J Am Coll Cardiol. 2008;52:1834-1843.

22. Goldenberg I, Kutyifa V, Klein HU, et al. Survival with cardiacresynchronization therapy in mild heart failure. NEngl J Med. 2014;370: 1694-1701.

23. Linde C, Gold MR, Abraham WT, et al. Long-term impact of cardiac resynchronization therapy in mild heart failure: 5 -year results from the resynchronization reverses remodeling in systolic left ventricular dysfunction (REVERSE) study. Eur Heart J. 2013;34:2592-2599.

24. Exner DV, Birnie DH, Moe G, et al. Canadian cardiovascular society guidelines on the use of cardiac resynchronization therapy: evidence and patient selection. Can J Cardiol. 2013;29:182-195.

25. Tracy CM, Epstein AE, Darbar D, et al. 2012 ACCF/AHA/HRS focused update of the 2008 guidelines for device-based therapy of cardiac rhythm abnormalities: a report of the American College of Cardiology Foundation/American Heart Association Task Force on Practice Guidelines. Heart Rhythm. 2012;9:1737-1753.

26. Thibault B, Harel F, Ducharme A, et al. Cardiac resynchronization therapy in patients with heart failure and a QRS complex $<120$ milliseconds: The evaluation of resynchronization therapy for heart failure (LESSEREARTH) trial. Circulation. 2013;127:873-881.

27. Zareba W, Klein H, Cygankiewicz I, et al. Effectiveness of cardiac resynchronization therapy by QRS morphology in the multicenter automatic defibrillator implantation trial-cardiac resynchronization therapy (MADIT-CRT). Circulation. 2011;123:1061-1072.

28. Sipahi I, Carrigan TP, Rowland DY, Stambler BS, Fang JC. Impact of qrs duration on clinical event reduction with cardiac resynchronization therapy: meta-analysis of randomized controlled trials. Arch Intern Med. 2011;171:1454-1462.

29. Sipahi I, Chou JC, Hyden M, et al. Effect of QRS morphology on clinical event reduction with cardiac resynchronization therapy: meta-analysis of randomized controlled trials. Am Heart J. 2012;163:260-267.

30. Bilchick KC, Kamath S, DiMarco JP, Stukenborg GJ. Bundle-branch block morphology and other predictors of outcome after cardiac resynchronization therapy in medicare patients. Circulation. 2010;122:2022-2030.

31. Peterson PN, Greiner MA, Qualls LG, et al. QRS duration, bundle-branch block morphology, and outcomes among older patients with heart failure receiving cardiac resynchronization therapy. JAMA. 2013;310:617-626.

32. Miyazaki C, Powell BD, Bruce CJ, et al. Comparison of echocardiographic dyssynchrony assessment by tissue velocity and strain imaging in subjects with or without systolic dysfunction and with or without left bundle-branch block. Circulation. 2008;117:2617-2625.

33. Chung ES, Leon AR, Tavazzi L, et al. Results of the predictors of response to CRT (PROSPECT) trial. Circulation. 2008;117:2608-2616.

34. Yu CM, Sanderson JE, Gorcsan J 3rd. Echocardiography, dyssynchrony, and the response to cardiac resynchronization therapy. Eur Heart $J$. 2010;31:2326-2337.

35. Risum N, Williams ES, Khouri MG, et al. Mechanical dyssynchrony evaluated by tissue doppler cross-correlation analysis is associated with long-term survival in patients after cardiac resynchronization therapy. Eur Heart J. 2013;34:48-56.

36. Bax JJ, Gorcsan J 3rd. Echocardiography and noninvasive imaging in cardiac resynchronization therapy: results of the PROSPECT (predictors of response to cardiac resynchronization therapy) study in perspective. J Am Coll Cardiol. 2009;53:1933-1943.

37. Bleeker GB, Holman ER, Steendijk P, et al. Cardiac resynchronization therapy in patients with a narrow qrs complex. $J$ Am Coll Cardiol. 2006;48:2243-2250.

38. Yu CM, Chan YS, Zhang Q, et al. Benefits of cardiac resynchronization therapy for heart failure patients with narrow QRS complexes and coexisting systolic asynchrony by echocardiography. JAm Coll Cardiol. 2006; $48: 2251-2257$.

39. Beshai JF, Grimm RA, Nagueh SF, et al. Cardiac-resynchronization therapy in heart failure with narrow QRS complexes. $N$ Engl $J$ Med. 2007;357:2461-2471. 
40. Ruschitzka F, Abraham WT, Singh JP, et al. Cardiac-resynchronization therapy in heart failure with a narrow QRS complex. $N$ Engl J Med. 2013;369:1395-1405.

41. Wilton SB, Leung AA, Ghali WA, Faris P, Exner DV. Outcomes of cardiac resynchronization therapy in patients with versus those without atrial fibrillation: a systematic review and meta-analysis. Heart Rhythm. 2011;8:1088-1094.

42. Leclercq C, Walker S, Linde C, et al. Comparative effects of permanent biventricular and right-univentricular pacing in heart failure patients with chronic atrial fibrillation. Eur Heart J. 2002;23:1780-1787.

43. Brignole M, Botto G, Mont L, et al. Cardiac resynchronization therapy in patients undergoing atrioventricular junction ablation for permanent atrial fibrillation: a randomized trial. Eur Heart J. 2011;32:2420-2429.

44. Doshi RN, Daoud EG, Fellows C, et al. Left ventricular-based cardiac stimulation post AV nodal ablation evaluation (the PAVE study). J Cardiovasc Electrophysiol. 2005;16:1160-1165.

45. Gasparini M, Leclercq C, Lunati M, et al. Cardiac resynchronization therapy in patients with atrial fibrillation: the CERTIFY study (cardiac resynchronization therapy in atrial fibrillation patients multinational registry). JACC Heart Fail. 2013;1:500-507.

46. Ruwald AC, Pietrasik G, Goldenberg I, et al. The effect of intermittent atrial tachyarrhythmia on heart failure or death in cardiac resynchronization therapy with defibrillator versus implantable cardioverterdefibrillator patients: a MADIT-CRT substudy (multicenter automatic defibrillator implantation trial with cardiac resynchronization therapy). J Am Coll Cardiol. 2014;63:1190-1197.

47. Stockburger M, Gomez-Doblas JJ, Lamas G, et al. Preventing ventricular dysfunction in pacemaker patients without advanced heart failure: results from a multicentre international randomized trial (PREVENT-HF). Eur J Heart Fail. 2011;13:633-641.

48. Yu CM, Chan JY, Zhang Q, et al. Biventricular pacing in patients with bradycardia and normal ejection fraction. N Engl J Med. 2009;361: 2123-2134.

49. Curtis AB, Worley SJ, Adamson PB, et al. Biventricular pacing for atrioventricular block and systolic dysfunction. NEngl J Med. 2013;368: 1585-1593.

50. Funck RC, Blanc JJ, Mueller HH, et al. Biventricular stimulation to prevent cardiac desynchronization: rationale, design, and endpoints of the 'biventricular pacing for atrioventricular block to prevent cardiac desynchronization (BioPace)' study. Europace. 2006;8:629-635.

51. Funck RC, Mueller HH, Lunati M, et al. Characteristics of a large sample of candidates for permanent ventricular pacing included in the biventricular pacing for atrio-ventricular block to prevent cardiac desynchronization study (BioPace). Europace. 2014;16:354-362.

52. Singh JP, Klein HU, Huang DT, et al. Left ventricular lead position and clinical outcome in the multicenter automatic defibrillator implantation trial-cardiac resynchronization therapy (MADIT-CRT) trial. Circulation. 2011;123:1159-1166.

53. Thebault C, Donal E, Meunier C, et al. Sites of left and right ventricular lead implantation and response to cardiac resynchronization therapy observations from the reverse trial. Eur Heart J. 2012;33:2662-2671.

54. Kutyifa V, Zareba W, McNitt S, et al. Left ventricular lead location and the risk of ventricular arrhythmias in the MADIT-CRT trial. Eur Heart J. 2013;34:184-190.

55. Khan FZ, Virdee MS, Palmer CR, et al. Targeted left ventricular lead placement to guide cardiac resynchronization therapy: The TARGET study: a randomized, controlled trial. J Am Coll Cardiol. 2012;59:1509-1518.

56. Saba S, Marek J, Schwartzman D, et al. Echocardiography-guided left ventricular lead placement for cardiac resynchronization therapy: results of the speckle tracking assisted resynchronization therapy for electrode region trial. Circ Heart Fail. 2013;6:427-434.

57. Sommer A, Kronborg MB, Poulsen SH, et al. Empiric versus imaging guided left ventricular lead placement in cardiac resynchronization therapy (ImagingCRT): study protocol for a randomized controlled trial. Trials. 2013;14:113.
58. Ge Y, Ruwald AC, Kutyifa V, et al. A metric for evaluating the cardiac response to resynchronization therapy. Am J Cardiol. 2014;113: 1371-1377.

59. Becker M, Hoffmann R, Schmitz F, et al. Relation of optimal lead positioning as defined by three-dimensional echocardiography to long-term benefit of cardiac resynchronization. Am J Cardiol. 2007;100: 1671-1676.

60. Bleeker GB, Kaandorp TA, Lamb HJ, et al. Effect of posterolateral scar tissue on clinical and echocardiographic improvement after cardiac resynchronization therapy. Circulation. 2006;113:969-976.

61. Sade LE, Saba S, Marek JJ, et al. The association of left ventricular lead position related to regional scar by speckle-tracking echocardiography with clinical outcomes in patients receiving cardiac resynchronization therapy. J Am Soc Echocardiogr. 2014;27:648-656.

62. Delgado V, van Bommel RJ, Bertini M, et al. Relative merits of left ventricular dyssynchrony, left ventricular lead position, and myocardial scar to predict long-term survival of ischemic heart failure patients undergoing cardiac resynchronization therapy. Circulation. 2011;123:70-78.

63. Koplan BA, Kaplan AJ, Weiner S, et al. Heart failure decompensation and all-cause mortality in relation to percent biventricular pacing in patients with heart failure: is a goal of $100 \%$ biventricular pacing necessary? J Am Coll Cardiol. 2009;53:355-360.

64. Hayes DL, Boehmer JP, Day JD, et al. Cardiac resynchronization therapy and the relationship of percent biventricular pacing to symptoms and survival. Heart Rhythm. 2011;8:1469-1475.

65. Ruwald AC, Kutyifa V, Ruwald MH, et al. The association between biventricular pacing and cardiac resynchronization therapy-defibrillator efficacy when compared with implantable cardioverter defibrillator on outcomes and reverse remodelling. Eur Heart J. Epub August 11, 2014. DOI: http://dx.doi.org/10.1093/eurheartj/ehu294.

66. Ruwald MH, Mittal S, Ruwald AC, et al. Association between frequency of atrial and ventricular ectopic beats and biventricular pacing percentage and outcomes in patients with cardiac resynchronization therapy. J Am Coll Cardiol. 2014;64:971-981.

67. Ellenbogen KA, Gold MR, Meyer TE, et al. Primary results from the SmartDelay determined AV optimization: a comparison to other AV delay methods used in cardiac resynchronization therapy (SMART-AV) trial: a randomized trial comparing empirical, echocardiography-guided, and algorithmic atrioventricular delay programming in cardiac resynchronization therapy. Circulation. 2010;122:2660-2668.

68. Martin DO, Lemke B, Birnie D, et al. Investigation of a novel algorithm for synchronized left-ventricular pacing and ambulatory optimization of cardiac resynchronization therapy: results of the adaptive crt trial. Heart Rhythm. 2012;9:1807-1814.

69. Naqvi TZ. Echocardiography-guided biventricular pacemaker optimization. JACC Cardiovasc Imaging. 2010;3:1168-1180.

70. Rafie R, Naqvi TZ. Echocardiography-guided biventricular pacemaker optimization: role of echo doppler in hemodynamic assessment and improvement. Expert Rev Cardiovasc Ther. 2012;10:859-874.

71. Gorcsan J 3rd, Abraham T, Agler DA, et al. Association between frequency of atrial and ventricular ectopic beats and biventricular pacing percentage and outcomes in patients with cardiac resynchronization therapy. J Am Soc Echocardiogr. 2008;21:191-213.

72. Raphael CE, Kyriacou A, Jones S, et al. Multinational evaluation of the interpretability of the iterative method of optimisation of av delay for crt. Int J Cardiol. 2013;168:407-413.

73. Risum N, Sogaard P, Hansen TF, et al. Comparison of dyssynchrony parameters for vv-optimization in crt patients. Pacing Clin Electrophysiol. 2013;36:1382-1390.

74. Leon AR, Abraham WT, Brozena S, et al. Cardiac resynchronization with sequential biventricular pacing for the treatment of moderate-tosevere heart failure. J Am Coll Cardiol. 2005;46:2298-2304.

75. Thomas DE, Yousef ZR, Fraser AG. A critical comparison of echocardiographic measurements used for optimizing cardiac resynchronization therapy: stroke distance is best. Eur J Heart Fail. 2009;11:779-788.

76. Mortensen PT, Sogaard P, Mansour H, et al. Sequential biventricular pacing: evaluation of safety and efficacy. Pacing Clin Electrophysiol. 2004;27:339-345. 
77. Boriani G, Muller CP, Seidl KH, et al. Randomized comparison of simultaneous biventricular stimulation versus optimized interventricular delay in cardiac resynchronization therapy. The resynchronization for the hemodynamic treatment for heart failure management ii implantable cardioverter defibrillator (RHYTHM II ICD) study. Am Heart J. 2006;151:1050-1058.

78. Rao RK, Kumar UN, Schafer J, et al. Reduced ventricular volumes and improved systolic function with cardiac resynchronization therapy: a randomized trial comparing simultaneous biventricular pacing, sequential biventricular pacing, and left ventricular pacing. Circulation. 2007;115:2136-2144.

79. Gras D, Gupta MS, Boulogne E, Guzzo L, Abraham WT. Optimization of AV and VV delays in the real-world CRT patient population: an international survey on current clinical practice. Pacing Clin Electrophysiol. 2009;32 Suppl 1:S236-S239.

80. Whinnett ZI, Sohaib SM, Jones S, et al. British randomised controlled trial of AV and VV optimization ("BRAVO") study: rationale, design, and endpoints. BMC Cardiovasc Disord. 2014;14:42.

81. Schmidt S, Hurlimann D, Starck CT, et al. Treatment with higher dosages of heart failure medication is associated with improved outcome following cardiac resynchronization therapy. Eur Heart J. 2014;35:1051-1060.

82. Fung JW, Chan JY, Kum LC, et al. Suboptimal medical therapy in patients with systolic heart failure is associated with less improvement by cardiac resynchronization therapy. Int J Cardiol. 2007;115:214-219.

83. Penn J, Goldenberg I, McNitt S, et al. Changes in drug utilization and outcome in relation to the efficacy of cardiac resynchronization in the multicenter automatic defibrillator implantation trial with cardiac resynchronization therapy (MADIT-CRT). J Am Coll Cardiol. 2014;63:12S.

84. Heywood JT, Fonarow GC, Yancy CW, et al. Comparison of medical therapy dosing in outpatients cared for in cardiology practices with heart failure and reduced ejection fraction with and without device therapy: report from IMPROVE HF. Circ Heart Fail. 2010;3:596-605.

85. Voigt A, Shalaby A, Adelstein E, Saba S. Beta-blocker utilization and outcomes in patients receiving cardiac resynchronization therapy. Clin Cardiol. 2010;33:E1-E5.

86. Ruwald MH, Ruwald AC, Jons C, et al. Effect of metoprolol versus carvedilol on outcomes in madit-crt (multicenter automatic defibrillator implantation trial with cardiac resynchronization therapy). J Am Coll Cardiol. 2013;61:1518-1526.

87. Ruwald MH, Abu-Zeitone A, Jons C, et al. Impact of carvedilol and metoprolol on inappropriate implantable cardioverter-defibrillator therapy: the MADIT-CRT trial (multicenter automatic defibrillator implantation with cardiac resynchronization therapy). $J$ Am Coll Cardiol. 2013;62:1343-1350.

88. Mullens W, Grimm RA, Verga T, et al. Insights from a cardiac resynchronization optimization clinic as part of a heart failure disease management program. J Am Coll Cardiol. 2009;53:765-773.

89. Mullens W, Kepa J, De Vusser P, et al. Importance of adjunctive heart failure optimization immediately after implantation to improve longterm outcomes with cardiac resynchronization therapy. Am J Cardiol. 2011;108:409-415.

90. Altman RK, Parks KA, Schlett CL, et al. Multidisciplinary care of patients receiving cardiac resynchronization therapy is associated with improved clinical outcomes. Eur Heart J. 2012;33:2181-2188.
91. Alaeddini J, Wood MA, Amin MS, Ellenbogen KA. Gender disparity in the use of cardiac resynchronization therapy in the united states. Pacing Clin Electrophysiol. 2008;31:468-472.

92. Ector H, Vardas P. Current use of pacemakers, implantable cardioverter defibrillators, and resynchronization devices: data from the registry of the European Heart Rhythm Association. 2007;9:I44-I49.

93. Yao G, Freemantle N, Calvert MJ, et al. The long-term costeffectiveness of cardiac resynchronization therapy with or without an implantable cardioverter-defibrillator. Eur Heart J. 2007;28: $42-51$.

94. Lam SK, Owen A. Combined resynchronisation and implantable defibrillator therapy in left ventricular dysfunction: Bayesian network meta-analysis of randomised controlled trials. BMJ. 2007;335:925.

95. Barsheshet A, Wang PJ, Moss AJ, et al. Reverse remodeling and the risk of ventricular tachyarrhythmias in the MADIT-CRT (multicenter automatic defibrillator implantation trial-cardiac resynchronization therapy). J Am Coll Cardiol. 2011;57:2416-2423.

96. Ruwald MH, Solomon SD, Foster E, et al. Left Ventricular Ejection Fraction Normalization in Cardiac Resynchronization Therapy and Risk of Ventricular Arrhythmias and Clinical Outcomes: Results from the MADIT-CRT Trial. Circulation. Epub 2014 Oct 9.

97. Barsheshet A, Moss AJ, Huang DT, et al. Applicability of a risk score for prediction of the long-term (8-year) benefit of the implantable cardioverter-defibrillator. J Am Coll Cardiol. 2012;59: 2075-2079.

98. Bardy GH, Lee KL, Mark DB, et al. Amiodarone or an implantable cardioverter-defibrillator for congestive heart failure. $N \mathrm{Engl} \mathrm{J} \mathrm{Med}$. 2005;352:225-237

99. Goldenberg I, Moss AJ, Hall WJ, et al. Predictors of response to cardiac resynchronization therapy in the multicenter automatic defibrillator implantation trial with cardiac resynchronization therapy (MADIT-CRT). Circulation. 2011;124:1527-1536.

100. Danish Study Group. Danish ICD study in patients With dilated cardiomyopathy (DANISH). NLM identifier: NCT00542945.

101. Al-Majed NS, McAlister FA, Bakal JA, Ezekowitz JA. Meta-analysis: cardiac resynchronization therapy for patients with less symptomatic heart failure. Ann Intern Med. 2011;154:401-412.

102. Adabag S, Roukoz H, Anand IS, Moss AJ. Cardiac resynchronization therapy in patients with minimal heart failure: a systematic review and meta-analysis. J Am Coll Cardiol. 2011;58:935-941.

103. Udo EO, Zuithoff NP, van Hemel NM, et al. Incidence and predictors of short- and long-term complications in pacemaker therapy: the FOLLOWPACE study. Heart Rhythm. 2012;9:728-735.

104. Kirkfeldt RE, Johansen JB, Nohr EA, et al. Risk factors for lead complications in cardiac pacing: a population-based cohort study of 28,860 Danish patients. Heart Rhythm. 2011;8:1622-1628.

105. Kirkfeldt RE, Johansen JB, Nohr EA, et al. Pneumothorax in cardiac pacing: a population-based cohort study of 28,860 Danish patients Europace. 2012;14:1132-1138.

106. Kirkfeldt RE, Johansen JB, Nohr EA, Jorgensen OD, Nielsen JC. Complications after cardiac implantable electronic device implantations: an analysis of a complete, nationwide cohort in Denmark. Eur Heart J. 2014;35:1186-1194

Research Reports in Clinical Cardiology

\section{Publish your work in this journal}

Research Reports in Clinical Cardiology is an international, peerreviewed, open access journal publishing original research, reports, editorials, reviews and commentaries on all areas of cardiology in the clinic and laboratory. The manuscript management system is completely online and includes a very quick and fair peer-review system.

Visit http://www.dovepress.com/testimonials.php to read real quotes from published authors. 